\title{
creative
commons
}

\section{A note on extremal results on directed acyclic graphs}

\author{
Álvaro Martínez-Pérez * \\ Departamento de Análisis Económico y Finanzas, Universidad de Castilla-La Mancha, \\ Avda. Real Fábrica de Seda, s/n. 45600 Talavera de la Reina, Toledo, Spain

\section{Luis Montejano $^{\dagger}$, Deborah Oliveros ${ }^{\ddagger}$} \\ Instituto de Matemáticas, Universidad Nacional Autónoma de México, \\ Área de la Investigación Científica, Circuito Exterior, C.U., Coyoacán 04510, \\ México D.F., México
}

Received 17 May 2016, accepted 31 May 2017, published online 6 February 2018

\begin{abstract}
This paper studies the maximum number of edges of a Directed Acyclic Graph (DAG) with $n$ vertices in terms of it's longest path $\ell$. We prove that in general this number is the Turán number $t(n, l+1)$, the maximum number of edges in a graph with $n$ vertices without a clique of size $\ell+2$. Furthermore, we find the maximum number of edges in a DAG which is either reduced, strongly reduced or extremely reduced and we relate this extremal result with the family of intersection graphs of families of boxes with transverse intersection.
\end{abstract}

Keywords: Directed graphs, Turán numbers, intersection graphs of families of boxes.

Math. Subj. Class.: 05C20, 52C99

\section{Introduction}

One of the fundamental results in extremal graph theory is the Theorem of Turán (1941) which states that a graph with $n$ vertices that has more than $t(n, k)$ edges, will always contain a complete subgraph of size $k+1$. The Turán graph $T(n, k)$, is a $k$-partite graph on $n$ vertices whose partite sets are as nearly equal in cardinality, and has the property

\footnotetext{
* Partially supported by MTM 2015-63612P.

${ }^{\dagger}$ Supported by CONACyT 166306.

${ }_{\ddagger}$ Partially supported by PAPIIT 104915 and CONACyT 166306.

E-mail address: alvaro.martinezperez@uclm.es (Álvaro Martínez-Pérez), luis@matem.unam.mx (Luis Montejano),dolivero@matem.unam.mx (Deborah Oliveros)
} 
that contains the maximum posible number of edges $t(n, k)$ of any graph not containing a clique of size $k+1$. It is known that $t(n, k) \leq\left(1-\frac{1}{k}\right) \frac{n^{2}}{2}$, and equality holds if $k$ divides $n$. In fact, $\lim _{n \rightarrow \infty} \frac{t(n, m)}{n^{2} / 2}=1-\frac{1}{m}$. See [1].

Turán numbers for several families of graphs have been studied in the context of extremal graph theory, see for example [3] and [4]. In ([2, 7]) the authors analyze, among other things, the intersection graphs of boxes in $\mathbb{R}^{d}$ proving that, if $\mathcal{T}(n, k, d)$ denotes the maximal number of intersection pairs in a family $\mathcal{F}$ of $n$ boxes in $\mathbb{R}^{d}$ with the property that no $k+1$ boxes in $\mathcal{F}$ have a point in common (with $n \geq k \geq d \geq 1$ ), then $\mathcal{T}(n, k, d)=\mathcal{T}(n-k+d, d)+\mathcal{T}(n, k-d+1,1)$, with $\mathcal{T}(n, k, 1)=\left(\begin{array}{l}n \\ 2\end{array}\right)-\left(\begin{array}{c}n-k+1 \\ 2\end{array}\right)$ being the precise bound in dimension 1 for the family of interval graphs.

Turán numbers have played and important role for several variants of the Turán Theorem and its relation with the fractional Helly Theorem (see [5, 6]).

The purpose of this paper is to study the maximum number of edges in directed acyclic graphs with $n$ vertices with respect to it's longest path. That turns out to be related with the extremal behavior of the family of intersection graphs for a collection of boxes in $\mathbb{R}^{2}$ with transverse intersection.

The first result, Theorem 2.10, states that in a directed acyclic graph with $n$ vertices, if the longest path has length $\ell$, then the maximal number of edges is the Turán number $t(n, \ell+1)$.

Theorem 3.19 and its Corollaries state that given a directed acyclic graph $\vec{G}$ with $n$ vertices such that the longest path has length $\ell$, then if $\vec{G}$ is either reduced, strongly reduced or extremely reduced, $\vec{G}$ has at most $t(n-\ell+1,2)+\mathcal{T}(n, \ell, 1)$ edges, where again $\mathcal{T}(n, \ell, 1)$ denotes the maximal number of intersecting pairs in a family $\mathcal{F}$ of $n$ intervals in $\mathbb{R}$ with the property that no $\ell+1$ intervals in $\mathcal{F}$ have a point in common.

In fact, this bound is best possible. The bound is reached by the intersection graph of a collection of boxes in $\mathbb{R}^{2}$ with transverse intersection (see Proposition 4.6). This graph is extremely reduced (and thus is also strongly reduced and reduced, see Proposition 4.4).

\section{Directed acyclic graphs}

By a directed acyclic graph, DAG, we mean a simple directed graph without directed cycles. A DAG, $\vec{G}=(\mathcal{V}, \overrightarrow{\mathcal{E}})$, with vertex set $\mathcal{V}$ and directed edge set $\overrightarrow{\mathcal{E}}$ is transitive if for every $x, y, z \in \mathcal{V}$, if $\{x, y\},\{y, z\} \in \overrightarrow{\mathcal{E}}$ then $\{x, z\} \in \overrightarrow{\mathcal{E}}$.

Definition 2.1. A topological order of a directed graph $\vec{G}$ is an ordering $\mathcal{O}$ of its vertices $\left\{v_{1}, v_{2}, \ldots, v_{n}\right\}$ so that for every edge $\left\{v_{i}, v_{j}\right\}$ then $i<j$.

The following proposition is a well known result:

Proposition 2.2. A directed graph $\vec{G}$ is a DAG if and only if $\vec{G}$ has a topological order.

Given any set $X$, by $|X|$ we denote the cardinal of $X$.

Definition 2.3. The indegree, $\operatorname{deg}^{-}(v)$, of a vertex $v$ is the number of directed edges $\{x, v\}$ with $x \in \mathcal{V}$. The outdegree, $\operatorname{deg}^{+}(v)$, of a vertex $v$ is the number of directed edges $\{v, x\}$ with $x \in \mathcal{V}$. Notice that each directed edge $\{v, w\}$ adds one outdegree to the vertex $v$ and one indegree to the vertex $w$. Therefore, $\sum_{v \in \mathcal{V}} \operatorname{deg}^{+}(v)=\sum_{v \in \mathcal{V}} \operatorname{deg}^{-}(v)=|(\overrightarrow{\mathcal{E}})|$.

The degree of a vertex is $\operatorname{deg}(v)=\operatorname{deg}^{-}(v)+\operatorname{deg}^{+}(v)$. 
Definition 2.4. A vertex $v$ such that $\operatorname{deg}^{-}(v)=0$ is called source. A vertex $v$ such that $\operatorname{deg}^{+}(v)=0$ is called sink.

It is well known that every DAG $\vec{G}$ has at least one source and one sink.

Definition 2.5. Given a DAG, $\vec{G}=(\mathcal{V}, \overrightarrow{\mathcal{E}})$, a directed path $\vec{\gamma}$ in $G$ is a sequence of vertices $\left\{v_{0}, \ldots, v_{n}\right\}$ such that $\left\{v_{i-1}, v_{i}\right\} \in \overrightarrow{\mathcal{E}}$ for every $1 \leq i \leq n$. Here, $\vec{\gamma}$ has length $n$, and endpoint $v_{n}$.

Observe that since DAG's are acyclic, all the vertices on a directed path are different.

Definition 2.6. Given a DAG, $\vec{G}=(\mathcal{V}, \overrightarrow{\mathcal{E}})$, let $\Gamma: \mathcal{V} \rightarrow \mathbb{N}$ be such that $\Gamma(v)=k$ if there exists a directed path $\vec{\gamma}$ in $G$ of length $k$ with endpoint $v$ and there is no directed path $\vec{\gamma}^{\prime}$ with endpoint $v$ and length greater than $k$.

Definition 2.7. Given a DAG, $\vec{G}=(\mathcal{V}, \overrightarrow{\mathcal{E}})$ suppose that $\ell=\max \{k \mid \Gamma(v)=k$ for every $v \in \mathcal{V}\}$. Notice that, since $\vec{G}$ has no directed cycle, $\ell \leq|\mathcal{V}|$. Then, let us define a partition $P_{\Gamma}=\left\{V_{0}, \ldots, V_{\ell}\right\}$ of $\mathcal{V}$ such that $V_{i}:=\{v \in \mathcal{V} \mid \Gamma(v)=i\}$ for every $0 \leq i \leq \ell$.

Notice that $V_{0}$ is exactly the set of sources in $\vec{G}$ and $V_{\ell}$ is contained in the set of sinks in $G$.

Lemma 2.8. $V_{i}$ is nonempty for every $0 \leq i \leq \ell$.

Proof. Let $\left\{v_{0}, \ldots, v_{\ell}\right\}$ be a directed path of maximal length in $\vec{G}$. Clearly, for every $0 \leq i \leq \ell, v_{i} \notin V_{j}$ if $j<i$. Suppose $v_{i} \in V_{j}$ with $i<j \leq \ell$. Then, there is a directed path $\left\{v_{0}^{\prime}, \ldots, v_{j}^{\prime}=v_{i}\right\}$ with $j>i$ and $\left\{v_{0}^{\prime}, \ldots, v_{j}^{\prime}, v_{i+1}, \ldots, v_{\ell}\right\}$ is a directed path with length $j+l-i>\ell$ which contradicts the hypothesis.

Lemma 2.9. The induced subgraph with vertices $V_{i}, G\left[V_{i}\right]$, is independent (has no edges) for every $i$.

Proof. Let $v_{i}, v_{i}^{\prime} \in V_{i}$ and suppose $\left\{v_{i}, v_{i}^{\prime}\right\} \in \overrightarrow{\mathcal{E}}$. Let $\left\{v_{0}, \ldots, v_{i}\right\}$ be a path of length $i$ with endpoint $v_{i}$. Then, $\left\{v_{0}, \ldots, v_{i}, v_{i}^{\prime}\right\}$ defines a directed path of length $i+1$ which contradicts the fact that $v_{i}^{\prime} \in V_{i}$.

Recall that $T(n, \ell)$ denote the $\ell$-partite Turán graph with $n$ vertices and $t(n, \ell)$ denote the number of edges of $T(n, \ell)$.

Theorem 2.10. Let $\vec{G}=(\mathcal{V}, \overrightarrow{\mathcal{E}})$ be a DAG with $n$ vertices such that the longest directed path has length $\ell$. Then, $\vec{G}$ has at most $t(n, \ell+1)$ edges.

Proof. Consider the partition $P_{\Gamma}=\left\{V_{0}, \ldots, V_{\ell}\right\}$ of $\mathcal{V}$. By Lemma 2.9, this defines an $(\ell+1)$-partite directed graph. Thus, neglecting the orientation we obtain a complete $(\ell+1)$ partite graph with partition sets $V_{0}, \ldots, V_{\ell}$. Therefore, the number of edges is at most $t(n, \ell+1)$.

Remark 2.11. It is readily seen that the bound in Theorem 2.10 is best possible. Consider the Turán graph $T(n, \ell+1)$ and any ordering of the $\ell+1$ independent sets $V_{0}, \ldots, V_{\ell}$. Then, for every edge $\left\{v_{i}, v_{j}\right\}$ in $T(n, \ell)$ with $v_{i} \in V_{i}, v_{j} \in V_{j}$ and $i<j$ let us assume the orientation $\left\{v_{i}, v_{j}\right\}$. It is trivial to check that the resulting graph is a DAG with $t(n, \ell+1)$ edges. 


\section{Reduced, strongly reduced and extremely reduced DAGs}

Let $\mathcal{O}$ be a topological ordering in a DAG $\vec{G}$. Given any two vertices $v, w$, and two directed paths in $\vec{G}, \gamma, \gamma^{\prime}$, from $v$ to $w$, let us define $\gamma \cup_{\mathcal{O}} \gamma^{\prime}$ as the sequence of vertices defined by the vertices in $\gamma \cup \gamma^{\prime}$ in the order given by $\mathcal{O}$. Of course, this need not be, in general, a directed path from $v$ to $w$.

Let $\Gamma(u, v)$ be the set of all directed paths from $u$ to $v$. Let $\cup_{\mathcal{O}}\{\gamma \mid \gamma \in \Gamma(u, v)\}$ represent the sequence of all the vertices from the paths in $\Gamma(u, v)$ ordered according to $\mathcal{O}$.

Definition 3.1. A finite DAG $\vec{G}$ is strongly reduced if for any topological ordering $\mathcal{O}$ of $\vec{G}$, every pair of vertices, $v, w$, and every pair of directed paths, $\gamma, \gamma^{\prime}$, from $v$ to $w$, then $\gamma \cup_{\mathcal{O}} \gamma^{\prime}$ defines a directed path from $v$ to $w$.

Remark 3.2. Let $\vec{G}$ be DAG. Given any two vertices $v, w$, and two directed paths in $\vec{G}$, $\gamma, \gamma^{\prime}$, from $v$ to $w$, let us define $\gamma \leq \gamma^{\prime}$ if every vertex in $\gamma$ is also in $\gamma^{\prime}$. Clearly, " $\leq$ " is a partial order.

Definition 3.3. A vertex $w$ is reachable from a vertex $v$ if there is a directed path from $v$ to $w$.

Proposition 3.4. Given a finite $D A G \vec{G}=(\mathcal{V}, \overrightarrow{\mathcal{E}})$, the following properties are equivalent:

i) For every pair of vertices $v, w$ and every pair of paths, $\gamma, \gamma^{\prime}$, from $v$ to $w$, there exists a directed path from $v$ to $w, \gamma^{\prime \prime}$, such that $\gamma, \gamma^{\prime} \leq \gamma^{\prime \prime}$.

ii) For every pair of vertices $v, w$ such that $w$ is reachable from $v$, there is a directed path from $v$ to $w, \gamma_{M}$, such that for every directed path, $\gamma$, from $v$ to $w, \gamma \leq \gamma_{M}$.

iii) For every topological ordering $\mathcal{O}$ of $\vec{G}$ and any pair of vertices $v, w, \cup_{\mathcal{O}}\{\gamma \mid \gamma \in$ $\Gamma(u, v)\}$ defines a directed path from $v$ to $w$.

Proof. Since the graph is finite and the relation ' $\leq$ ' is transitive, $i$ ) and ii) are trivially equivalent.

If $i i)$ is satisfied, then it is trivial to see that $\cup_{\mathcal{O}}\{\gamma \mid \gamma \in \Gamma(u, v)\}=\gamma_{M}$ and iii) is satisfied. Also, it is readily seen that iii) implies ii) taking $\gamma_{M}:=\cup_{\mathcal{O}}\{\gamma \mid \gamma \in \Gamma(u, v)\}$.

Definition 3.5. We say that a finite DAG $\vec{G}$ is reduced if it satisfies any of the properties from Proposition 3.4.

Proposition 3.6. If a finite $D A G \vec{G}$ is strongly reduced, then $\vec{G}$ is reduced.

Proof. Since the graph is finite, it is immediate to see that being strongly reduced implies iii).

Remark 3.7. The converse is not true. The graph in the left from Figure 1 is clearly reduced. Notice that the directed path $\gamma_{M}:=\left\{v_{1}, v_{2}, v_{3}, v_{4}, v_{5}\right\}$ is an upper bound for every directed path from $v_{1}$ to $v_{5}$. However, if we consider the directed paths $\gamma=\left\{v_{1}, v_{2}, v_{5}\right\}$ and $\gamma^{\prime}=\left\{v_{1}, v_{4}, v_{5}\right\}$ with the topological order $\mathcal{O}=\left\{v_{1}, v_{2}, v_{3}, v_{4}, v_{5}\right\}$, then $\gamma \cup_{\mathcal{O}} \gamma^{\prime}=$ $\left\{v_{1}, v_{2}, v_{4}, v_{5}\right\}$ which is not a directed path. 

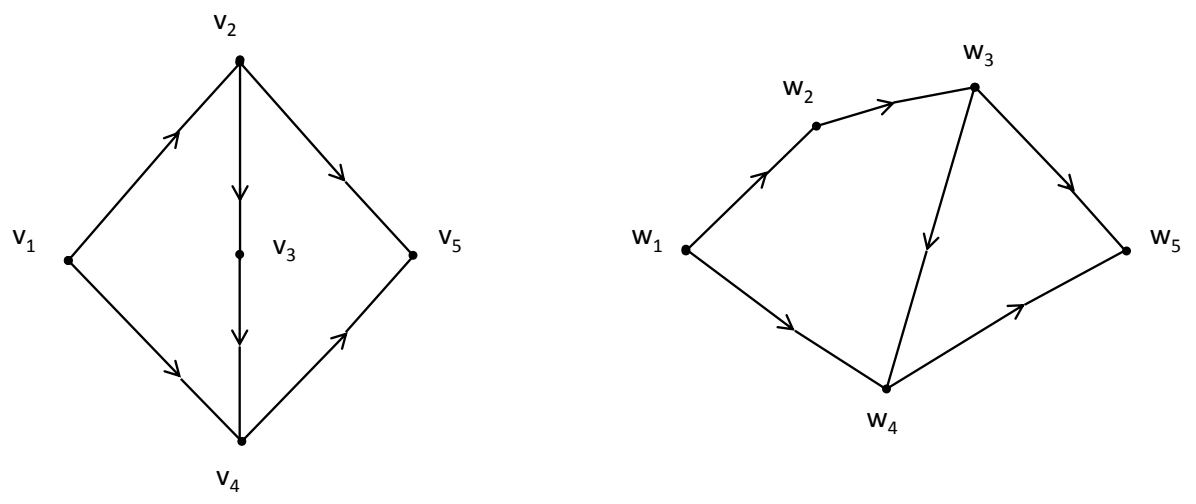

Figure 1: Being reduced does not imply being strongly reduced and being strongly reduced does not imply being extremely reduced.

Definition 3.8. Given a finite DAG $\vec{G}$ and a vertex $v \in \mathcal{V}$ we say that $w$ is an ancestor of $v$ if there is a directed path $\left\{w=v_{0}, \ldots, v_{k}=v\right\}$ and $w$ is a descendant of $v$ if there is a directed path $\left\{v=v_{0}, \ldots, v_{k}=w\right\}$.

Definition 3.9. We say that a finite DAG $\vec{G}$ is extremely reduced if for every pair of nonadjacent vertices $x, y$, if $x, y$ have a common ancestor, then they do not have a common descendant.

Proposition 3.10. If a DAG $\vec{G}=(\mathcal{V}, \overrightarrow{\mathcal{E}})$ is extremely reduced, then it is strongly reduced.

Proof. Let $\gamma=\left\{v, v_{1}, \ldots, v_{n}, w\right\}$ and $\gamma^{\prime}=\left\{v, w_{0}, \ldots, w_{m}, w\right\}$ be two directed paths in $\vec{G}$ from $v$ yo $w$. Let $\mathcal{O}$ be any topological order in $\vec{G}$ and consider $\gamma \cup_{\mathcal{O}} \gamma^{\prime}=\left\{v, z_{1}, \ldots, z_{k}\right.$, $w\}$. First, notice that $z_{1}$ is either $v_{1}$ or $w_{1}$. Therefore, $\left\{v, z_{1}\right\} \in \overrightarrow{\mathcal{E}}$. Also, $z_{k}$ is either $v_{n}$ or $w_{m}$, and $\left\{z_{k}, w\right\} \in \overrightarrow{\mathcal{E}}$. Now, for every $1<i \leq k$, let us see that $\left\{z_{i-1}, z_{i}\right\} \in \overrightarrow{\mathcal{E}}$. If $z_{i-1}, z_{i} \in \gamma$ or $z_{i-1}, z_{i} \in \gamma^{\prime}$, then they are consecutive vertices in a directed path and we are done. Otherwise, since $z_{i-1}, z_{i}$ have a common ancestor $v$ and a common descendant $w$, then there is a directed edge joining them and, since $z_{i-1}, z_{i}$ are sorted by a topological order, $\left\{z_{i-1}, z_{i}\right\} \in \overrightarrow{\mathcal{E}}$.

Remark 3.11. The converse is not true. The graph in the right from Figure 1 is strongly reduced. However, vertices $w_{2}$ and $w_{4}$ are not adjacent and have a common ancestor and a common descendent.

Proposition 3.12. If $\vec{G}$ is transitive, then the following properties are equivalent:

- $\vec{G}$ is extremely reduced,

- $\vec{G}$ is strongly reduced,

- $\vec{G}$ is reduced.

Proof. By Proposition 3.10 if $\vec{G}$ is extremely reduced, then it is strongly reduced. By Proposition 3.6, if $\vec{G}$ is strongly reduced, then it is reduced.

Suppose $\vec{G}$ is reduced and suppose that two vertices $x, y$ have a common ancestor, $v$, and a common descendant, $w$. Then, there are two directed paths $\gamma, \gamma^{\prime}$ from $v$ to $w$ such 
that $x \in \gamma$ and $y \in \gamma^{\prime}$. By property $i$ ) in Proposition 3.4, there exists a path $\gamma^{\prime \prime}$ in $\vec{G}$ from $v$ to $w$ such that $\gamma, \gamma^{\prime} \leq \gamma^{\prime \prime}$. In particular, $x, y \in \gamma^{\prime \prime}$. Therefore, either $x$ is reachable from $y$ or $y$ is reachable from $x$ in $\vec{G}$. Since $\vec{G}$ is transitive, this implies that $x, y$ are adjacent. Therefore, $\vec{G}$ is extremely reduced.

Definition 3.13. Given a DAG $\vec{G}=(\mathcal{V}, \overrightarrow{\mathcal{E}})$, the graph with vertex set $\mathcal{V}$ and edge set $\overrightarrow{\mathcal{E}^{\prime}}:=\overrightarrow{\mathcal{E}} \cup\{\{v, w\} \mid w$ is reachable from $v\}$ is called the transitive closure of $\vec{G}, T[\vec{G}]$.

It is immediate to check the following:

Proposition 3.14. Given any $D A G \vec{G}, T[\vec{G}]$ is transitive.

Proposition 3.15. If a DAG $\vec{G}$ is reduced, then the transitive closure $T[\vec{G}]$ is also reduced.

Proof. Suppose $\vec{G}$ satisfies $i$ ) in Proposition 3.4 and let $\gamma=\left\{v=v_{0}, \ldots, v_{n}=w\right\}$, $\gamma^{\prime}=\left\{v=w_{0}, \ldots, w_{m}=w\right\}$ be any pair of paths from $v$ to $w$ in $T[\vec{G}]$. Therefore, $v_{i}$ is reachable from $v_{i-1}$ in $\vec{G}$ for every $1 \leq i \leq n$ and $w_{i}$ is reachable from $w_{i-1}$ in $\vec{G}$ for every $1 \leq i \leq m$. Thus, there exist a path $\gamma_{0}$ in $\vec{G}$ such that $\gamma \leq \gamma_{0}$ and a path $\gamma_{0}^{\prime}$ in $\vec{G}$ such that $\gamma^{\prime} \leq \gamma_{0}^{\prime}$. By property $i$ ), there is a directed path from $v$ to $w$ such that $\gamma_{0}, \gamma_{0}^{\prime} \leq \gamma_{0}^{\prime \prime}$. Therefore, $\gamma, \gamma^{\prime} \leq \gamma_{0}^{\prime \prime}$ and $T[\vec{G}]$ satisfies $i$ ).

Then, from Propositions 3.6, 3.10, 3.12, 3.14 and 3.15:

Corollary 3.16. If a DAG $\vec{G}$ is reduced, then the transitive closure $T[\vec{G}]$ is extremely reduced and strongly reduced. In particular, if $\vec{G}$ is extremely reduced or strongly reduced, then $T[\vec{G}]$ is extremely reduced and strongly reduced.

Let us recall that

$$
\mathcal{T}(n, \ell, 1)=\left(\begin{array}{l}
n \\
2
\end{array}\right)-\left(\begin{array}{c}
n-\ell+1 \\
2
\end{array}\right)=(n-\ell+1)(\ell-1)+\frac{(\ell-1)(\ell-2)}{2}
$$

As it was proved in [7]:

Lemma 3.17. For $n \geq \ell$ and $d \geq 1$,

$$
\mathcal{T}(n+d, \ell, 1)-\mathcal{T}(n, \ell, 1)=d(\ell-1) .
$$

In particular, $\mathcal{T}(n+2, \ell, 1)-\mathcal{T}(n, \ell, 1)=2(\ell-1)$.

Also, from [7]:

Lemma 3.18. For $1 \leq d \leq n$,

$$
t(n+d, d)-t(n, d)=(d-1) n+\left(\begin{array}{l}
d \\
2
\end{array}\right)
$$

In particular, $t(n+2,2)-t(n, 2)=n+1$.

Theorem 3.19. Let $\vec{G}=(\mathcal{V}, \overrightarrow{\mathcal{E}})$ be a $D A G$ with $n$ vertices and such that the longest directed path has length $\ell \geq 1$. If $\vec{G}$ is extremely reduced, then $\vec{G}$ has at most $t(n-\ell+1,2)+$ $\mathcal{T}(n, \ell, 1)$ edges. 
Proof. Let us prove the result by induction on $n$. Suppose that the longest directed path has length $\ell$.

First, let us see that the result is true for $n=\ell+1$ and $n=\ell+2$.

If $n=\ell+1$ then $\vec{G}$ has at most $\frac{\ell(\ell+1)}{2}=\frac{(\ell-2)(\ell-1)}{2}+2(\ell-1)+1=\mathcal{T}(n, \ell, 1)+$ $t(n-\ell+1,2)$ edges. The last equation follows immediately from (3.1) and the fact that $t(2,2)=1$.

If $n=\ell+2$ then there are $\ell+1$ vertices which define a directed path $\gamma=\left\{v_{0}, \ldots, v_{\ell}\right\}$ and one vertex $w$ such that neither $\left\{w, v_{0}\right\}$ nor $\left\{v_{\ell}, w\right\}$ is a directed edge. Then, the partition $P_{\Gamma}=\left\{V_{0}, \ldots, V_{\ell}\right\}$ of $\vec{G}$ satisfies that $v_{i} \in V_{i}$ for every $0 \leq i \leq \ell$. Also, $w \in V_{j}$ for some $0 \leq j \leq \ell$ and $\left\{w, v_{j}\right\},\left\{v_{j}, w\right\}$ are not directed edges. Hence, $\operatorname{deg}(w) \leq \ell$. Therefore, $\vec{G}$ has at most $\frac{\ell(\ell+1)}{2}+\ell=\frac{(\ell-2)(\ell-1)}{2}+3(\ell-1)+2=\mathcal{T}(n, \ell, 1)+t(n-\ell+1,2)$ edges. The last equation follows immediately from (3.1) and the fact that $t(3,2)=2$.

Suppose the induction hypothesis holds when the graph has $n$ vertices and let $\#(\mathcal{V})=$ $n+2$. Also, by Proposition 3.15 we may assume that the graph is transitive.

Consider the partition $P_{\Gamma}=\left\{V_{0}, \ldots, V_{\ell}\right\}$ of $\mathcal{V}$. Let $\#\left(V_{i}\right)=r_{i}$. Let $v \in V_{0}$ and $w$ be any sink of $\vec{G}$. Consider any pair of vertices $v_{i}, v_{i}^{\prime} \in V_{i}$. Since $\vec{G}$ is extremely reduced and every two vertices in $V_{i}$ are non-adjacent, $v_{i}, v_{i}^{\prime}$ can not be both descendants of $v$ and ancestors of $w$ simultaneously. Hence, the number of edges joining the sets $\{v, w\}$ and $V_{i}$ are at most $r_{i}+1$. Therefore, there are at most $n+\ell-1$ edges joining $\{v, w\}$ and $G \backslash\{v, w\}$

Since $G \backslash\{v, w\}$ has $n$ vertices, by hypothesis, it contains at most $t(n-\ell+1,2)+$ $\mathcal{T}(n, \ell, 1)$ edges.

Finally, there is at most 1 edge in the subgraph induced by $\{v, w\}$.

Therefore, by Lemmas 3.17 and 3.18, $|\vec{E}(G)| \leq t(n-\ell+1,2)+\mathcal{T}(n, \ell, 1)+n+\ell=$ $t(n-\ell+3,2)+\mathcal{T}(n+2, \ell, 1)$.

By Corollary 3.16 we know that the extremal graph for reduced and strongly reduced graphs is transitive. Thus, from Theorem 3.19 and Proposition 3.12 we obtain the following.

Corollary 3.20. Let $\vec{G}=(\mathcal{V}, \overrightarrow{\mathcal{E}})$ be a DAG with $n$ vertices and such that the longest directed path has length $\ell \geq 1$. If $\vec{G}$ is reduced, then $\vec{G}$ has at most $t(n-\ell+1,2)+$ $\mathcal{T}(n, \ell, 1)$ edges.

Corollary 3.21. Let $\vec{G}=(\mathcal{V}, \overrightarrow{\mathcal{E}})$ be a DAG with $n$ vertices and such that the longest directed path has length $\ell \geq 1$. If $\vec{G}$ is strongly reduced, then $\vec{G}$ has at most $t(n-\ell+$ $1,2)+\mathcal{T}(n, \ell, 1)$ edges.

\section{Directed intersection graphs of boxes}

Definition 4.1. Let $\mathcal{R}$ be a collection of boxes with parallel axes in $\mathbb{R}^{2}$. Let $\vec{G}=(\mathcal{V}, \overrightarrow{\mathcal{E}})$ be a directed graph such that $\mathcal{V}=\mathcal{R}$ and given $R, R^{\prime} \in \mathcal{R}$ with $R=I \times J, R^{\prime}=I^{\prime} \times J^{\prime}$ then $\left\{R, R^{\prime}\right\} \in \overrightarrow{\mathcal{E}}$ if and only if $I \subset I^{\prime}$ and $J^{\prime} \subset J$ (i.e. there is an edge if and only if the intersection is transverse and the order is defined by the subset relation in the first coordinate). Let us call $\vec{G}$ the directed intersection graph of $\mathcal{R}$.

Definition 4.2. Let $\mathcal{R}$ be a collection of boxes with parallel axes in $\mathbb{R}^{2}$. We say that $\mathcal{R}$ is a collection with transverse intersection if for every pair of boxes either they are disjoint or their intersection is transverse. 


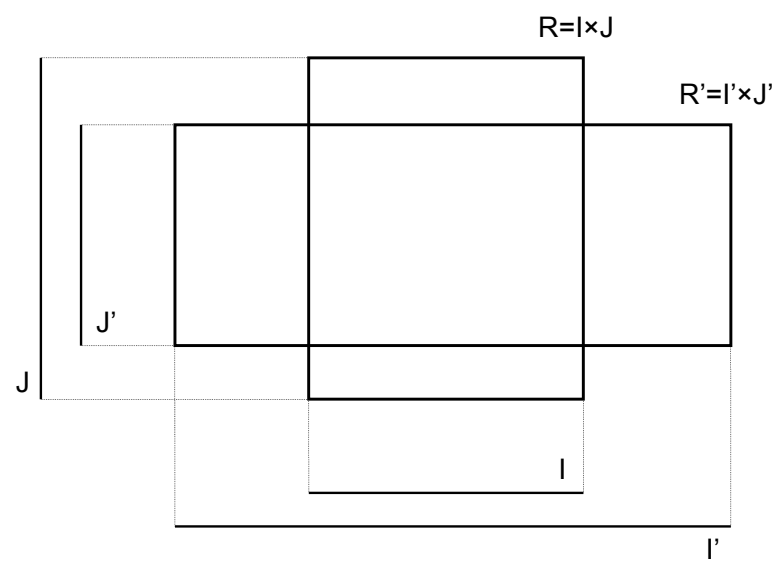

Figure 2: The transverse intersection above induces a directed edge $\left\{R, R^{\prime}\right\}$.

Proposition 4.3. Let $\mathcal{R}$ be a collection of boxes with parallel axes in $\mathbb{R}^{2}$ and $\vec{G}$ be the induced directed intersection graph. If two vertices $v, w$ have both a common ancestor and a common descendant in $\vec{G}$, then the corresponding boxes $R_{v}, R_{w}$ intersect.

Proof. Let $a$ be a common ancestor and $R_{a}=I_{a} \times J_{a}$ be the corresponding box. Let $b$ be a common descendant and $R_{b}=I_{b} \times J_{b}$ be the corresponding box. Then if $R_{v}=$ $I_{v} \times J_{v}, R_{w}=I_{w} \times J_{w}$ are the boxes corresponding to $v$ and $w$ respectively, it follows by construction that $I_{a} \subset I_{v}, I_{w}$ and $J_{b} \subset J_{v}, J_{w}$. Therefore, $I_{a} \times J_{b} \subset R_{v}, R_{w}$ and $R_{v} \cap R_{w} \neq \emptyset$.

Proposition 4.4. If $\mathcal{R}$ is a collection of boxes with parallel axes in $\mathbb{R}^{2}$ with transverse intersection, then the induced directed intersection graph $G$ is extremely reduced and transitive.

Proof. First notice that the transitivity holds simply by the transverse intersection property. Let $v, w$ be two vertices such that there is no edge joining them. This means, by construction, that their corresponding boxes do not have a transverse intersection. Since $\mathcal{R}$ has transverse intersection, this implies that these boxes do not intersect. Thus, by Proposition 4.3, if $v, w$ have a common ancestor, then they can not have a common descendant.

Remark 4.5. Consider the bipartite graph $G$ from Figure 3 with the partition given by \{letters, numbers\} and assume all directed edges go from letters into numbers. Note that $G$ is extremely reduced, transitive and acyclic. Notice that the induced subgraphs given by the sets $C_{1}:=\{1,2, A, B\}, C_{2}:=\{3,4, C, D\}$ and $C_{3}:=\{5,6, E, F\}$ are three cycles of length 4 . Furthermore the induced subgraph given by the set of vertices $\{1,2,3,4,8,9$, $A, B, C, D, H, I\}$ is realizable as boxes in $\mathbb{R}^{2}$ (see Figure 4) note, that contains $C_{1}$ and $C_{2}$ and its realization force one of them to be inside the other say $C_{1}$ inside $C_{2}$. Similarly the induced subgraphs given by the set of vertices $\{1,2,5,6, A, B, E, F, 7,12, G, L\}$ and the set of vertices $\{3,4,5,6, C, D, E, F, 10,11, J, K\}$ forces necessarily a system of tree squares one inside the other. However, intervals given by $\{7,8,9,10,11,12\}$ and $\{G, H, I, J, K, L\}$ are forced to have more intersections that those given by the graph. In 


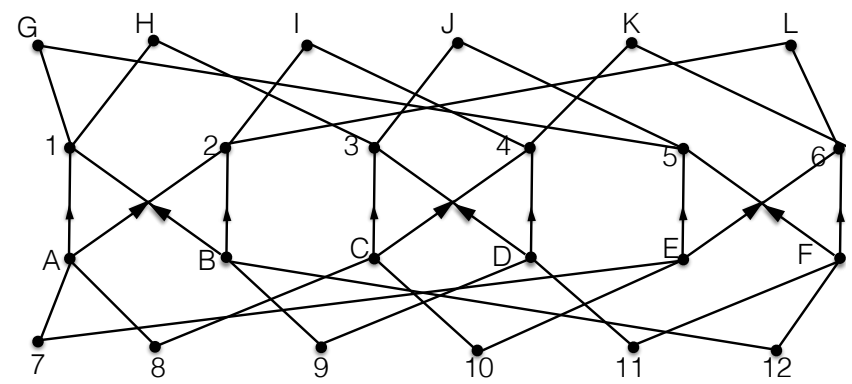

Figure 3: The bipartite, transitive, and extremely reduced DAG, $G$ with partition given by \{letters, numbers $\}$ and edges directed from letters into numbers. This graph is not realizable as a family of boxes in $\mathbb{R}^{2}$.

other words, there is no family of boxes (or intervals) that realizes such a graph or for which it is induced the graph $G$. Then, the converse of Proposition 4.4 is not true.

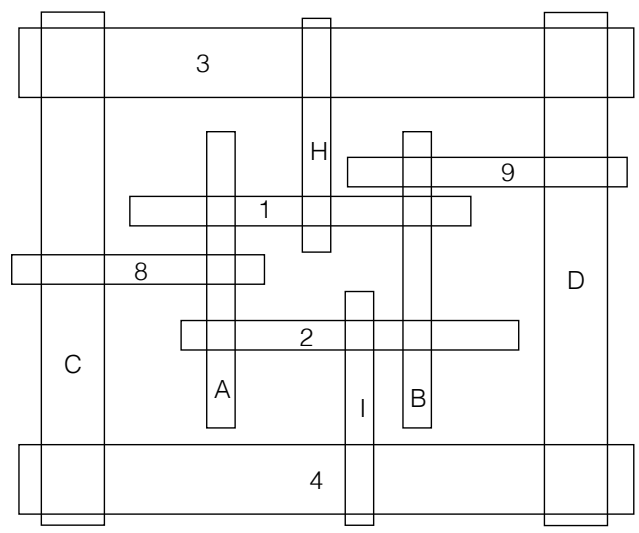

Figure 4: Realization in $\mathbb{R}^{2}$ of the induced subgraph with vertices $\{1,2,3,4,8,9, A$, $B, C, D, H, I\}$ of the graph shown in Figure 3 .

Let $G[r, l, s]$ be the graph, $G(\mathcal{V}, \overrightarrow{\mathcal{E}})$, such that:

- $\mathcal{V}=\left\{x_{1}, \ldots, x_{r}, y_{1}, \ldots, y_{l-1}, z_{1}, \ldots, z_{s}\right\}$

- $\left\{x_{i}, x_{j}\right\} \notin \overrightarrow{\mathcal{E}}$ for any $i \neq j$,

- $\left\{z_{i}, z_{j}\right\} \notin \overrightarrow{\mathcal{E}}$ for any $i \neq j$,

- $\left\{x_{i}, y_{j}\right\} \in \overrightarrow{\mathcal{E}}$ for every $i, j$,

- $\left\{y_{i}, y_{j}\right\} \in \overrightarrow{\mathcal{E}}$ for every $i<j$,

- $\left\{y_{i}, z_{j}\right\} \in \overrightarrow{\mathcal{E}}$ for every $i, j$,

- $\left\{x_{i}, z_{j}\right\} \in \overrightarrow{\mathcal{E}}$ for every $i, j$.

This is the directed intersection graph from the collection of boxes in Figure 5. 

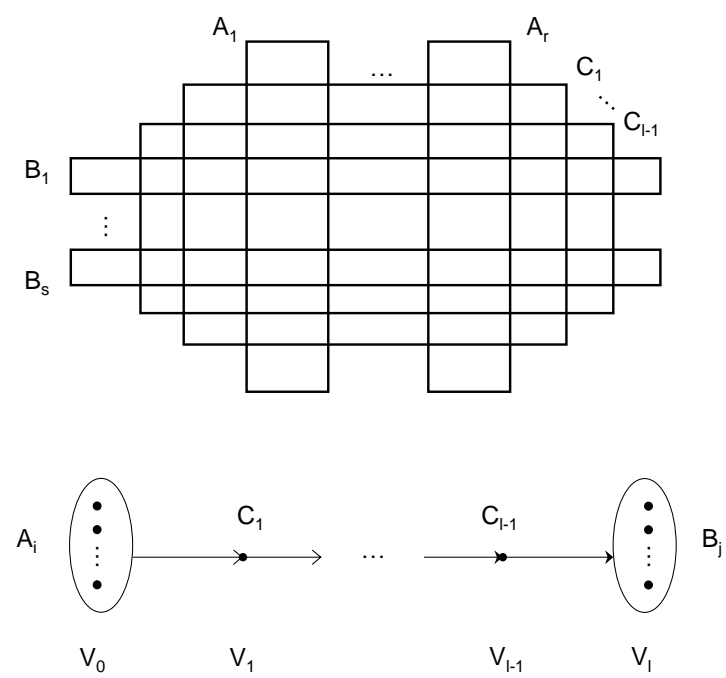

Figure 5: The graph $G[r, l, s]$ corresponds to the directed intersection graph of the collection in the figure where $x_{i} \sim A_{i}, y_{j} \sim C_{j}$ and $z_{k} \sim B_{k}$. Notice that the graph is transitive although not every edge is represented in the figure.

By Proposition 4.4, $G[r, l, s]$ is a transitive extremely reduced DAG. In particular, $G[r, l, s]$ is strongly reduced and reduced.

Now, to prove that the bound obtained in Theorem 3.19 and its corollaries is best possible, it is immediate to check the following:

Proposition 4.6. If $n-\ell$ is even, $G\left[\frac{n-\ell}{2}, \ell, \frac{n-\ell}{2}\right]$ has $t(n-\ell+1,2)+\mathcal{T}(n, \ell, 1)$ edges. If $n-\ell$ is odd, $G\left[\frac{n-\ell+1}{2}, \ell, \frac{n-\ell-1}{2}\right]$ has $t(n-\ell+1,2)+\mathcal{T}(n, \ell, 1)$ edges.

\section{References}

[1] M. Aigner and G. M. Ziegler, Proofs from THE BOOK, Springer-Verlag, Berlin, 1998, doi: 10.1007/978-3-662-22343-7.

[2] I. Bárány, F. Fodor, Á. Martínez-Pérez, L. Montejano, D. Oliveros and A. Pór, A fractional Helly theorem for boxes, Comput. Geom. 48 (2015), 221-224, doi:10.1016/j.comgeo.2014.09.007.

[3] B. Bollobás, Extremal Graph Theory, Dover Publications, Mineola, New York, 2004.

[4] R. Diestel, Graph Theory, volume 173 of Graduate Texts in Mathematics, Springer-Verlag, Berlin, 3rd edition, 2005, http: / / diestel-graph-theory.com/.

[5] G. Kalai, Intersection patterns of convex sets, Israel J. Math. 48 (1984), 161-174, doi:10.1007/ bf02761162.

[6] M. Katchalski and A. Liu, A problem of geometry in $\mathbf{R}^{n}$, Proc. Amer. Math. Soc. 75 (1979), 284-288, doi:10.2307/2042758.

[7] Á. Martínez-Pérez, L. Montejano and D. Oliveros, Extremal results on intersection graphs of boxes in $\mathbb{R}^{d}$, in: K. Adiprasito, I. Bárány and C. Vîlcu (eds.), Convexity and Discrete Geometry Including Graph Theory, Springer, volume 148 of Springer Proceedings in Mathematics \& Statistics, pp. 137-144, 2016, doi:10.1007/978-3-319-28186-5_11, papers from the conference held in Mulhouse, September 7 - 11, 2014. 